\title{
Small prime solutions of ternary linear equations
}

\author{
by \\ JiAnYA LiU (Jinan) and KAI-MAN Tsang (Hong Kong)
}

1. Introduction. For any integer $n$, we consider the ternary linear equation

$$
a_{1} p_{1}+a_{2} p_{2}+a_{3} p_{3}=n
$$

where $p_{j}$ are prime variables and the coefficients $a_{j}$ are non-zero integers. A necessary condition for the solubility of (1.1) is

$$
a_{1}+a_{2}+a_{3} \equiv n \bmod 2 .
$$

We also suppose

$$
\left(a_{i}, a_{j}\right)=1, \quad\left(a_{j}, n\right)=1 \quad \text { for } 1 \leq i<j \leq 3,
$$

and write $A=\max \left\{2,\left|a_{1}\right|,\left|a_{2}\right|,\left|a_{3}\right|\right\}$. The main result of this paper is the following.

Theorem 1.1. Assume (1.2) and (1.3).

(i) If $a_{1}, a_{2}, a_{3}$ are not all of the same sign, then (1.1) has solutions in primes $p_{j}$ satisfying

$$
\left|a_{j}\right| p_{j} \ll|n|+A\left|a_{1} a_{2} a_{3}\right|^{5 / 2} \log ^{26} A .
$$

(ii) If $a_{1}, a_{2}, a_{3}$ are all positive, then (1.1) is soluble whenever

$$
n \gg A\left(a_{1} a_{2} a_{3}\right)^{5 / 2} \log ^{26} A .
$$

It follows from the above theorem that, in case (i), (1.1) has prime solutions satisfying $p_{j} \ll|n|+A^{15 / 2} \log ^{26} A$, and in case (ii), (1.1) is soluble in primes $p_{j}$ if $n \gg A^{17 / 2} \log ^{26} A$.

This problem was first raised and investigated by Baker in his well known work [1]. In the case when condition (1.3) is relaxed to that any three of $a_{1}, a_{2}, a_{3}, n$ are relatively prime, the problem was settled qualitatively by

2000 Mathematics Subject Classification: 11P32, 11P05, 11P55.

The first author supported by HKSAR-RGC Grant HKU7028/03P and NSF Grant \#10125101.

The second author supported by HKSAR-RGC Grant HKU7028/03P. 
M. C. Liu and Tsang [10]. In Choi [2] the bound $A^{4190}$ was obtained in place of those in our Theorem 1.1. The number 4190 was subsequently reduced to 45 by M. C. Liu and Wang [12], and then to 38 by Li [8]. Under the Generalized Riemann Hypothesis, Choi, M. C. Liu, and Tsang [3] reduced the constant to $5+\varepsilon$.

We prove our theorem by the circle method, and the idea will be explained in $\S 2$. At this stage, we point out that in contrast to the earlier works [2], [10], [11], [12], which treated the enlarged major arcs by the Deuring-Heilbronn phenomenon, we show that under the stronger condition (1.3), the possible existence of Siegel's zero does not have special influence and hence the Deuring-Heilbronn phenomenon can be avoided. This observation enables us to get better results without using heavy numerical computations.

Notation. As usual, $\varphi(n), \mu(n)$ and $\Lambda(n)$ stand for the functions of Euler, Möbius and von Mangoldt respectively, and $\tau(n)$ is the divisor function. We use $\chi \bmod q$ and $\chi^{0} \bmod q$ to denote a Dirichlet character and the principal character modulo $q$, and $L(s, \chi)$ is the Dirichlet $L$-function. $r \sim R$ means $R<r \leq 2 R$. The letters $c$ and $c_{j}$ denote absolute positive constants, but the value of $c$ without subscript may vary at different places. The letter $\varepsilon$ denotes a positive constant which is arbitrarily small.

2. Outline of the method. Denote by $r(n)$ the weighted number of solutions of (1.1), that is

$$
r(n)=\sum_{\substack{n=a_{1} p_{1}+a_{2} p_{2}+a_{3} p_{3} \\ M<\left|a_{j}\right| p_{j} \leq N}}\left(\log p_{1}\right)\left(\log p_{2}\right)\left(\log p_{3}\right),
$$

where $M=N / 200$. We will estimate $r(n)$ by the circle method. To this end, we set

$$
P=(N / A)^{2 / 5}, \quad L=\log N, \quad Q=N /\left(P L^{2}\right) .
$$

By Dirichlet's lemma on rational approximation, each $\alpha \in[1 / Q, 1+1 / Q]$ may be written in the form

$$
\alpha=a / q+\lambda, \quad|\lambda| \leq 1 /(q Q),
$$

for some integers $a, q$ with $1 \leq a \leq q \leq Q$ and $(a, q)=1$. We denote by $\mathfrak{M}(q, a)$ the set of $\alpha$ satisfying $(2.2)$ and write $\mathfrak{M}$ for the union of all these major arcs, that is, those $\mathfrak{M}(q, a)$ with $1 \leq a \leq q \leq P$ and $(a, q)=1$. It follows from $2 P \leq Q$ that these major $\operatorname{arcs} \mathfrak{M}(a, q)$ are mutually disjoint. Define, as usual, the minor arcs $\mathfrak{m}$ to be the complement of $\mathfrak{M}$ in $[1 / Q, 1+1 / Q]$. Let 


$$
S_{j}(\alpha)=\sum_{M<\left|a_{j}\right| p \leq N}(\log p) e\left(a_{j} p \alpha\right) .
$$

Then we have

$$
r(n)=\int_{1 / Q}^{1+1 / Q} S_{1}(\alpha) S_{2}(\alpha) S_{3}(\alpha) e(-n \alpha) d \alpha=\int_{\mathfrak{M}}+\int_{\mathfrak{m}} .
$$

The integral over the major arcs $\mathfrak{M}$ causes the main difficulty, which is handled by the following.

Theorem 2.1. Assume (1.3). Let $P, Q$ be defined by (2.1). Then

$$
\int_{\mathfrak{M}} S_{1}(\alpha) S_{2}(\alpha) S_{3}(\alpha) e(-n \alpha) d \alpha=\mathfrak{S}(n, P) \mathfrak{I}(n)+O\left(\frac{N^{2}}{\left|a_{1} a_{2} a_{3}\right| L}\right),
$$

where $\mathfrak{S}(n, P)$ and $\mathfrak{I}(n)$ are defined in $(2.5)$ and (2.6) respectively.

The proof of this theorem forms the bulk of this paper, in $\S \S 3-5$. The quality of our bounds in Theorem 1.1 depends on the size of our major arcs which, as can be seen in (2.1), are quite large. The key observation is that under the assumption (1.3), we can save one negative power of $r_{0}$ in Lemma 3.1 below. With this saving, (2.4) can be derived from a hybrid estimate for Dirichlet polynomials (Lemma 3.4 below), Heath-Brown's identity, Gallagher's lemma, and classical results on the distribution of the zeros of $L$-functions.

To derive Theorem 1.1 from Theorem 2.1, we need to bound $\mathfrak{S}(n, P)$ and $\mathfrak{I}(n)$ from below. For $\chi \bmod q$, define

$$
C(\chi, a)=\sum_{h=1}^{q} \bar{\chi}(h) e\left(\frac{a h}{q}\right), \quad C(q, a)=C\left(\chi^{0}, a\right) .
$$

If $\chi_{1}, \chi_{2}, \chi_{3}$ are characters modulo $q$, we write

$$
\begin{gathered}
B\left(q, \chi_{1}, \chi_{2}, \chi_{3}\right)=\sum_{\substack{h=1 \\
(h, q)=1}}^{q} e\left(-\frac{h n}{q}\right) C\left(\chi_{1}, a_{1} h\right) C\left(\chi_{2}, a_{2} h\right) C\left(\chi_{3}, a_{3} h\right), \\
B(q)=B\left(q, \chi^{0}, \chi^{0}, \chi^{0}\right), \quad A(q)=\frac{B(q)}{\varphi^{3}(q)} .
\end{gathered}
$$

Note that the functions $B(q)$ and $A(q)$ depend also on $a_{1}, a_{2}, a_{3}$ and $n$, which are fixed throughout, but we do not make this explicit for simplicity. Finally, define

$$
\mathfrak{S}(n, x)=\sum_{q \leq x} A(q)
$$

The following two results are Lemmas 4.4 and 7.2 of [10]. 
Lemma 2.2. Assuming (1.2), we have $\mathfrak{S}(n, P) \geq c_{1}$ for some absolute constant $c_{1}>0$.

Lemma 2.3. Suppose (1.3) and

(i) the $a_{j}$ 's are not all of the same sign and $N \geq 3|n|$; or

(ii) the $a_{j}$ 's are all positive and $N=n$.

Then

$$
\Im(n):=\sum_{\substack{a_{1} m_{1}+a_{2} m_{2}+a_{3} m_{3}=n \\ M<\left|a_{j}\right| m_{j} \leq N}} 1 \asymp \frac{N^{2}}{\left|a_{1} a_{2} a_{3}\right|} .
$$

We now derive Theorem 1.1 from Theorem 2.1 and Lemmas 2.2 and 2.3.

Proof of Theorem 1.1. We start from (2.3) and let $N_{j}=N /\left|a_{j}\right|$. To estimate the integral over $\mathfrak{m}$, we appeal to Lemma 7.1 in [10]:

$$
S_{3}(\alpha) \ll L^{4}\left(N_{3} P^{-1 / 2}\left|a_{3}\right|^{1 / 2}+N_{3}^{4 / 5}+N_{3}^{1 / 2} Q^{1 / 2}\right) \ll N L^{4} / \sqrt{\left|a_{3}\right| P} .
$$

Also, we have the following mean-value estimate:

$$
\int_{1 / Q}^{1+1 / Q}\left|S_{j}(\alpha)\right|^{2} d \alpha \ll L N_{j},
$$

which in combination with Schwarz's inequality gives

$$
\int_{1 / Q}^{1+1 / Q}\left|S_{1}(\alpha) S_{2}(\alpha)\right| d \alpha \ll \frac{L N}{\sqrt{\left|a_{1} a_{2}\right|}} .
$$

It therefore follows from (2.7) and (2.8) that

$$
\left|\int_{\mathfrak{m}}\right| \ll \frac{N^{2} L^{5}}{\sqrt{\left|a_{1} a_{2} a_{3}\right| P}} .
$$

The contribution from the major arcs is estimated in Theorem 2.1 and, together with (2.9), gives

$$
r(n)=\mathfrak{S}(n, P) \mathfrak{I}(n)+O\left(\frac{N^{2}}{\left|a_{1} a_{2} a_{3}\right| L}+\frac{N^{2} L^{5}}{\sqrt{\left|a_{1} a_{2} a_{3}\right| P}}\right) .
$$

With conditions (i) or (ii) in Lemma 2.3, we deduce from Lemmas 2.2, 2.3 and the above formula that

$$
r(n) \gg \frac{N^{2}}{\left|a_{1} a_{2} a_{3}\right|}
$$

provided that $P \gg L^{10.4}\left|a_{1} a_{2} a_{3}\right|$, or equivalently, $N \gg A L^{26}\left|a_{1} a_{2} a_{3}\right|^{5 / 2}$. This proves Theorem 1.1. 
3. Proof of Theorem 2.1: preliminaries. In this section, we give four lemmas pertaining to the proof of Theorem 2.1.

LEMMA 3.1. Let $q$ and $m$ be positive integers.

(i) If $\chi \bmod q$ is a character, then

$$
|C(\chi, m)| \leq(q, m)^{1 / 2} q^{1 / 2} .
$$

(ii) Let $\chi_{j} \bmod r_{j}$ with $j=1,2,3$ be primitive characters, $r_{0}=\left[r_{1}, r_{2}, r_{3}\right]$, and $\chi^{0}$ be the principal character modulo $q$. Then

$$
\sum_{\substack{q \leq x \\ r_{0} \mid q}} \frac{1}{\varphi^{3}(q)}\left|B\left(q, \chi_{1} \chi^{0}, \chi_{2} \chi^{0}, \chi_{3} \chi^{0}\right)\right| \ll \frac{r_{0}^{2}}{\varphi^{3}\left(r_{0}\right)} \sqrt{\left(\varpi, r_{0}\right)} \log ^{2} x ;
$$

here and throughout $\varpi=\left|a_{1} a_{2} a_{3} n\right|$.

Proof. (i) Let $\chi \bmod q$ be induced by the primitive character $\chi^{*} \bmod q^{*}$. Write $q=q_{1} q_{2}$ with $\left(q_{2}, q^{*}\right)=1$ and $p\left|q_{1} \Rightarrow p\right| q^{*}$. Then, by [6, p. 450],

$$
C(\chi, m)=\chi^{*}\left(\frac{m}{(m, q)}\right) \bar{\chi}^{*}\left(\frac{q}{q^{*}(m, q)}\right) \mu\left(\frac{q}{q^{*}(m, q)}\right) \frac{\varphi(q)}{\varphi(q /(m, q))} C\left(\chi^{*}, 1\right)
$$

if $q^{*}=q_{1} /\left(q_{1}, m\right)$; otherwise $C(\chi, m)=0$.

We first establish our assertion in the special case that $q$ is a power of a prime, say $q=p^{\alpha}$. In this case we must have $q_{1}=p^{\alpha}$ and $q_{2}=1$. Let $q^{*}=p^{\beta}$ and $p^{\gamma} \| m$. We may suppose $\gamma \leq \alpha$, since otherwise $p$ divides $m /(m, q)$ and hence

$$
\chi^{*}\left(\frac{m}{(m, q)}\right)=0,
$$

which gives $C(\chi, m)=0$. Also, we only have to consider the case $q^{*}=$ $q_{1} /\left(q_{1}, m\right)$, that is,

$$
\beta=\alpha-\gamma,
$$

since otherwise we have $C(\chi, m)=0$ again. Finally, we have

$$
|C(\chi, m)| \leq \frac{\varphi\left(p^{\alpha}\right)}{\varphi\left(p^{\alpha-\gamma}\right)}\left|C\left(\chi^{*}, 1\right)\right| \leq p^{\gamma+\beta / 2}=p^{\gamma / 2+\alpha / 2}=(q, m)^{1 / 2} q^{1 / 2} .
$$

This proves our assertion in the special case $q=p^{\alpha}$.

The general case can be established by decomposing $C(\chi, m)$ according to the canonical factorization of $\chi \bmod q$ and then using the Chinese remainder theorem.

(ii) By Lemma 4.5 in [10], we have

$$
B\left(q, \chi_{1} \chi^{0}, \chi_{2} \chi^{0}, \chi_{3} \chi^{0}\right)=B\left(r_{0}, \chi_{1} \chi^{0}, \chi_{2} \chi^{0}, \chi_{3} \chi^{0}\right) B\left(q / r_{0}\right)
$$

if $\left(r_{0}, q / r_{0}\right)=1$; and it vanishes otherwise. Here we note that the moduli of the principal characters in the above functions $B(\cdot)$ on the right hand side 
are $r_{0}$ and $q / r_{0}$ respectively. It therefore follows that

$$
\begin{aligned}
& \sum_{\substack{q \leq x \\
r_{0} \mid q}} \frac{1}{\varphi^{3}(q)}\left|B\left(q, \chi_{1} \chi^{0}, \chi_{2} \chi^{0}, \chi_{3} \chi^{0}\right)\right| \\
& =\frac{1}{\varphi^{3}\left(r_{0}\right)}\left|B\left(r_{0}, \chi_{1} \chi^{0}, \chi_{2} \chi^{0}, \chi_{3} \chi^{0}\right)\right| \sum_{\substack{q \leq x / r_{0} \\
\left(r_{0}, q\right)=1}}|A(q)| .
\end{aligned}
$$

Now the argument of Lemma 4.4(1) in [10] gives

$$
\sum_{q \leq x}|A(q)| \ll \log ^{2} x
$$

It remains, therefore, to estimate $B\left(r_{0}, \chi_{1} \chi^{0}, \chi_{2} \chi^{0}, \chi_{3} \chi^{0}\right)$.

By the definition of $C(\chi, m)$, we have $C(\chi, m h)=\chi(h) C(\chi, m)$. Consequently,

$$
\begin{aligned}
B\left(r_{0}, \chi_{1} \chi^{0}, \chi_{2} \chi^{0}, \chi_{3} \chi^{0}\right) & \\
= & \sum_{\substack{h=1 \\
\left(h, r_{0}\right)=1}}^{r_{0}} e\left(-\frac{n h}{r_{0}}\right) C\left(\chi_{1} \chi^{0}, a_{1} h\right) C\left(\chi_{2} \chi^{0}, a_{2} h\right) C\left(\chi_{3} \chi^{0}, a_{3} h\right) \\
& =C\left(\chi_{1} \chi^{0}, a_{1}\right) C\left(\chi_{2} \chi^{0}, a_{2}\right) C\left(\chi_{3} \chi^{0}, a_{3}\right) C\left(\bar{\chi}_{1} \bar{\chi}_{2} \bar{\chi}_{3} \chi^{0},-n\right) .
\end{aligned}
$$

Now (i) and (1.3) gives

$$
\left|B\left(r_{0}, \chi_{1} \chi^{0}, \chi_{2} \chi^{0}, \chi_{3} \chi^{0}\right)\right| \leq r_{0}^{2} \sqrt{\left(a_{1}, r_{0}\right)\left(a_{2}, r_{0}\right)\left(a_{3}, r_{0}\right)\left(n, r_{0}\right)}=r_{0}^{2} \sqrt{\left(\varpi, r_{0}\right)},
$$

which together with (3.2) and (3.1) yields the desired result.

Recall $N_{j}=N /\left|a_{j}\right|$ for $j=1,2,3$, and set

$$
M_{j}=\frac{M}{\left|a_{j}\right|}, \quad V_{j}(\lambda)=\sum_{M<\left|a_{j}\right| m \leq N} e\left(a_{j} m \lambda\right),
$$

and

$$
W_{j}(\chi, \lambda)=\sum_{M<\left|a_{j}\right| p \leq N}(\log p) \chi(p) e\left(a_{j} p \lambda\right)-\delta_{\chi} \sum_{M<\left|a_{j}\right| m \leq N} e\left(a_{j} m \lambda\right)
$$

where $\delta_{\chi}=1$ or 0 according as $\chi$ is principal or not. Define

$$
J_{j}(R)=\sum_{r \sim R} \frac{\sqrt{(\varpi, r)}}{r} \sum_{\chi \bmod r}^{*} \max _{|\lambda| \leq 1 /(r Q)}\left|W_{j}(\chi, \lambda)\right|,
$$

and for any positive integer $g$,

$$
K_{j}(g, R)=\sum_{r \sim R} \frac{\sqrt{(\varpi,[g, r])}}{[g, r]} \sum_{\chi \bmod r}^{*}\left\{\int_{|\lambda| \leq 1 /(r Q)}\left|W_{j}(\chi, \lambda)\right|^{2} d \lambda\right\}^{1 / 2},
$$


where $\sum_{\chi \bmod r}^{*}$ is over all the primitive characters modulo $r$. To prove our Theorem 2.1, we need the following two key lemmas which will be proved in $\S 5$.

Lemma 3.2. For $P, Q$ satisfying (2.1), we have

(i) $K_{j}(g, R) \ll g^{-1} \sqrt{(\varpi, g)} \tau(g) \tau(\varpi) \sqrt{N}\left|a_{j}\right|^{-1} L^{c}$ if $R \ll P$,

(ii) $K_{j}(g, R) \ll g^{-1} \sqrt{(\varpi, g)} \tau(g) \sqrt{N}\left|a_{j}\right|^{-1} L^{c}$ if $R \ll N^{1 / 10}$.

Lemma 3.3. Let $P, Q$ be as in (2.1). We have

(i) $J_{j}(R) \ll \tau(\varpi) R^{-1 / 4} N\left|a_{j}\right|^{-1} L^{c}$ if $R \ll P$,

(ii) $J_{j}(R) \ll R^{-1 / 4} N\left|a_{j}\right|^{-1} L^{-c_{2}}$ for any large constant $c_{2}$ if $R \ll N^{1 / 10}$.

These two lemmas depend on a hybrid estimate for Dirichlet polynomials (Lemma 3.4 below). Let $X^{2 / 5}<Y \leq X$ and $D_{1}, \ldots, D_{10}$ be positive integers such that

$$
2^{-10} Y \leq D_{1} \cdots D_{10}<X \quad \text { and } \quad 2 D_{6}, \ldots, 2 D_{10} \leq X^{1 / 5} .
$$

For $j=1, \ldots, 10$, define

$$
b_{j}(m)= \begin{cases}\log m & \text { if } j=1, \\ 1 & \text { if } j=2, \ldots, 5, \\ \mu(m) & \text { if } j=6, \ldots, 10,\end{cases}
$$

where $\mu(n)$ is the Möbius function. For any Dirichlet character $\chi$ and complex variable $s$, define the functions

$$
f_{j}(s, \chi)=\sum_{m \sim D_{j}} \frac{b_{j}(m) \chi(m)}{m^{s}}
$$

and

$$
F_{\mathbf{D}}(s, \chi)=f_{1}(s, \chi) \cdots f_{10}(s, \chi),
$$

where $\mathbf{D}=\left(D_{1}, \ldots, D_{10}\right)$. The following hybrid estimate for $F_{\mathbf{D}}(s, \chi)$ is Lemma 2.1 in [9]. The parameter $d$ in (3.9) is crucial for our iterative argument in $\S 4$.

Lemma 3.4. Let $F_{\mathbf{D}}(s, \chi)$ be defined as above. Then for any $1 \leq R \leq X^{2}$ and $T>0$,

$$
\begin{aligned}
\sum_{\substack{r \sim R \\
d \mid r}} \sum_{\chi \bmod r}^{*} \int_{T}^{2 T} \mid F_{\mathbf{D}}( & \left.\frac{1}{2}+i t, \chi\right) \mid d t \\
& \ll\left\{\frac{R^{2}}{d} T+\frac{R}{d^{1 / 2}} T^{1 / 2} X^{3 / 10}+X^{1 / 2}\right\} \log ^{c} X .
\end{aligned}
$$


4. Proof of Theorem 2.1: an iterative method. Introducing Dirichlet characters, we can express the exponential sum $S_{j}(\alpha)$ as (see for example $[4, \S 26,(2)])$

$S_{j}\left(\frac{h}{q}+\lambda\right)=\frac{C\left(q, a_{j} h\right)}{\varphi(q)} V_{j}(\lambda)+\frac{1}{\varphi(q)} \sum_{\chi \bmod q} C\left(\chi, a_{j} h\right) W_{j}(\chi, \lambda)=: T_{j}+U_{j}$, say. Thus,

$$
\int_{\mathfrak{M}} S_{1}(\alpha) S_{2}(\alpha) S_{3}(\alpha) e(-n \alpha) d \alpha=I_{0}+I_{1}+I_{2}+I_{3},
$$

where $I_{0}, I_{1}, I_{2}, I_{3}$ are the contributions from, respectively,

$$
\begin{aligned}
& T_{1} T_{2} T_{3}, \\
& U_{1} T_{2} T_{3}+T_{1} U_{2} T_{3}+T_{1} T_{2} U_{3}, \\
& U_{1} U_{2} T_{3}+U_{1} T_{2} U_{3}+T_{1} U_{2} U_{3}, \\
& U_{1} U_{2} U_{3} .
\end{aligned}
$$

We shall now show that $I_{0}$ contains the main term for $\int_{\mathfrak{M}}$ and $I_{1}, I_{2}, I_{3}$ constitute the error term.

Lemma 4.1. For $j=1,2,3$, we have

(i) $\quad \int\left|V_{j}(\lambda)\right|^{2} d \lambda \ll N\left|a_{j}\right|^{-2}$, $|\lambda| \leq 1 /\left|2 a_{j}\right|$

(ii) $\quad \int\left|V_{j}(\lambda)\right|^{2} d \lambda \ll N\left|a_{j}\right|^{-1}$.

$|\lambda| \leq 1 / 2$

Proof. By definition of $V_{j}(\lambda)$,

$$
\int_{|\lambda| \leq 1 /\left|2 a_{j}\right|}\left|V_{j}(\lambda)\right|^{2} d \lambda=\sum_{M_{j}<m, m^{\prime} \leq N_{j}} \int_{|\lambda| \leq 1 /\left|2 a_{j}\right|} e\left(\left(m-m^{\prime}\right) a_{j} \lambda\right) d \lambda \ll \frac{N_{j}}{\left|a_{j}\right|} .
$$

Part (ii) follows from (i) and the fact that $V_{j}(\lambda)$ has period $\left|a_{j}\right|^{-1}$.

Lemma 4.2. For $(2 A)^{-1}<|\lambda|<1 / 2$, we have

$$
\max _{j=1,2,3}\left(\left\|a_{j} \lambda\right\|\right) \geq 1 /(2 A) .
$$

Proof. For $j=1,2,3$, let $\beta_{j}=\left\|a_{j} \lambda\right\|$ and $a_{j} \lambda=n_{j} \pm \beta_{j}$, where $n_{j}$ are integers. If the three rational numbers $n_{j} / a_{j}$ are all the same, then since $\left(a_{1}, a_{2}, a_{3}\right)=1$ they must be all equal to an integer $k$. Furthermore $k$ must be equal to zero, for otherwise, from

$$
\lambda=\frac{n_{1}}{a_{1}} \pm \frac{\beta_{1}}{a_{1}}=k \pm \frac{\beta_{1}}{a_{1}}
$$


we have $|\lambda| \geq|k|-1 / 2 \geq 1 / 2$, which is a contradiction. Hence

$$
\beta_{j}=\left|a_{j} \lambda\right|>\frac{\left|a_{j}\right|}{2 A}, \quad j=1,2,3 .
$$

This yields the desired bound.

On the other hand, if the three rational numbers $n_{j} / a_{j}$ are not all the same, $n_{1} / a_{1} \neq n_{2} / a_{2}$, say, then

$$
\left|\frac{\beta_{1}}{a_{1}} \pm \frac{\beta_{2}}{a_{2}}\right|=\left|\frac{n_{1}}{a_{1}}-\frac{n_{2}}{a_{2}}\right| \geq \frac{1}{\left|a_{1} a_{2}\right|} .
$$

Hence

$$
\max \left(\beta_{1}, \beta_{2}\right)\left(\frac{1}{\left|a_{1}\right|}+\frac{1}{\left|a_{2}\right|}\right) \geq \frac{1}{\left|a_{1} a_{2}\right|} .
$$

The desired bound again follows in this case.

By definition, $I_{0}$ is equal to

$$
\sum_{q \leq P} \frac{B(q)}{\varphi^{3}(q)} \int_{|\lambda| \leq 1 /(q Q)} V_{1}(\lambda) V_{2}(\lambda) V_{3}(\lambda) e(-n \lambda) d \lambda .
$$

We begin by extending the above integral to the interval $[-1 / 2,1 / 2]$ in two steps. First, by the obvious bound $V_{j}(\lambda) \ll\left\|a_{j} \lambda\right\|^{-1}=\left|a_{j} \lambda\right|^{-1}$ for $|\lambda| \leq 1 /(2 A)$, we have

$$
\begin{aligned}
\int_{1 /(q Q)<|\lambda| \leq 1 /(2 A)} V_{1}(\lambda) V_{2}(\lambda) & V_{3}(\lambda) e(-n \lambda) d \lambda \\
\ll & \int_{1 /(q Q)<|\lambda| \leq 1 /(2 A)} \frac{d \lambda}{\left|a_{1} a_{2} a_{3}\right| \lambda^{3}} \ll \frac{(q Q)^{2}}{\left|a_{1} a_{2} a_{3}\right|} .
\end{aligned}
$$

In view of (2.1) and (3.2), the error this contributes to $I_{0}$ in (4.2) is $\ll N^{2}\left(L\left|a_{1} a_{2} a_{3}\right|\right)^{-1}$, which is acceptable. Next, by the bound $V_{j}(\lambda) \ll$ $\left\|a_{j} \lambda\right\|^{-1}$ and Lemma 4.2, we have

$$
\begin{aligned}
& \int_{1 /(2 A)<|\lambda| \leq 1 / 2} V_{1}(\lambda) V_{2}(\lambda) V_{3}(\lambda) e(-n \lambda) d \lambda \\
& \ll A \sum_{1 \leq i<j \leq 3} \int_{|\lambda| \leq 1 / 2}\left|V_{i}(\lambda) V_{j}(\lambda)\right| d \lambda \ll A N,
\end{aligned}
$$

by Lemma 4.1(ii) and Schwarz's inequality. Clearly, the contribution of this to (4.2) is negligible. Hence we find that

$$
I_{0}=\sum_{q \leq P} \frac{B(q)}{\varphi^{3}(q)} \int_{|\lambda| \leq 1 / 2} V_{1}(\lambda) V_{2}(\lambda) V_{3}(\lambda) e(-n \lambda) d \lambda+O\left(\frac{N^{2}}{L\left|a_{1} a_{2} a_{3}\right|}\right) .
$$

In view of (2.6) and (2.5), this yields the main term on the right hand side of (2.4). 
We now turn to the terms in $I_{1}, I_{2}$, and $I_{3}$. The main feature in these terms is that each of them has at least one factor of $U_{j}$ and this is precisely the source of the saving of a factor $L^{-c_{2}}$ in Lemma 3.3(ii). We explain our strategy below by treating in detail the most complicated case, viz. $I_{3}$, and then indicate briefly the treatment for $I_{2}$ and $I_{1}$.

Reducing the characters in $I_{3}$ into primitive characters, we have

$$
\begin{aligned}
\left|I_{3}\right|= & \mid \sum_{q \leq P} \sum_{\chi_{1} \bmod q} \sum_{\chi_{2} \bmod q} \sum_{\chi_{3} \bmod q} \frac{B\left(q, \chi_{1}, \chi_{2}, \chi_{3}\right)}{\varphi^{3}(q)} \\
& \times \int_{|\lambda| \leq 1 /(q Q)} W_{1}\left(\chi_{1}, \lambda\right) W_{2}\left(\chi_{2}, \lambda\right) W_{3}\left(\chi_{3}, \lambda\right) e(-n \lambda) d \lambda \mid \\
\leq & \sum_{r_{i} \leq P} \sum_{\chi_{i} \bmod r_{i}}^{*} \sum_{\substack{q \leq P \\
r_{0} \mid q}} \frac{\left|B\left(q, \chi_{1} \chi^{0}, \chi_{2} \chi^{0}, \chi_{3} \chi^{0}\right)\right|}{\varphi^{3}(q)} \\
& \times \int_{|\lambda| \leq 1 /(q Q)}\left|W_{1}\left(\chi_{1} \chi^{0}, \lambda\right) W_{2}\left(\chi_{2} \chi^{0}, \lambda\right) W_{3}\left(\chi_{3} \chi^{0}, \lambda\right)\right| d \lambda,
\end{aligned}
$$

where

$$
\sum_{r_{i} \leq P}=\sum_{r_{1} \leq P} \sum_{r_{2} \leq P} \sum_{r_{3} \leq P}, \quad \sum_{\chi_{i} \bmod r_{i}}^{*}=\sum_{\chi_{1} \bmod r_{1}}^{*} \sum_{\chi_{2} \bmod r_{2}}^{*} \sum_{\chi_{3} \bmod r_{3}}^{*},
$$

$\chi^{0}$ is the principal character modulo $q$ and $r_{0}=\left[r_{1}, r_{2}, r_{3}\right]$. For $q \leq P$ and $M<\left|a_{j}\right| p \leq N$, we have $(q, p)=1$. Using this and (3.3), we see that $W_{j}\left(\chi_{j} \chi^{0}, \lambda\right)=W_{j}\left(\chi_{j}, \lambda\right)$ for the primitive characters $\chi_{j}$ above. Consequently, by Lemma 3.1(ii) we have

$$
\begin{aligned}
\left|I_{3}\right| \leq & \sum_{r_{i} \leq P} \sum_{\chi_{i} \bmod r_{i}|\lambda| \leq 1 /\left(r_{0} Q\right)}^{*}\left|W_{1}\left(\chi_{1}, \lambda\right) W_{2}\left(\chi_{2}, \lambda\right) W_{3}\left(\chi_{3}, \lambda\right)\right| d \lambda \\
& \times \sum_{\substack{q \leq P \\
r_{0} \mid q}} \frac{\left|B\left(q, \chi_{1} \chi^{0}, \chi_{2} \chi^{0}, \chi_{3} \chi^{0}\right)\right|}{\varphi^{3}(q)} \\
\ll & L^{3} \sum_{r_{i} \leq P} \frac{\sqrt{\left(\varpi, r_{0}\right)}}{r_{0}} \\
& \times \sum_{\chi_{i} \bmod r_{i}|\lambda| \leq 1 /\left(r_{0} Q\right)}^{*}\left|W_{1}\left(\chi_{1}, \lambda\right) W_{2}\left(\chi_{2}, \lambda\right) W_{3}\left(\chi_{3}, \lambda\right)\right| d \lambda \\
\ll & L^{6} \max _{R_{1}, R_{2}, R_{3} \leq P} I_{3}\left(R_{1}, R_{2}, R_{3}\right),
\end{aligned}
$$

where 


$$
\begin{aligned}
I_{3}\left(R_{1}, R_{2}, R_{3}\right) & =\sum_{r_{1} \sim R_{1}} \sum_{r_{2} \sim R_{2}} \sum_{r_{3} \sim R_{3}} \frac{\sqrt{\left(\varpi, r_{0}\right)}}{r_{0}} \\
& \times \sum_{\chi_{i} \bmod r_{i}|\lambda| \leq 1 /\left(r_{0} Q\right)}^{*} \int_{1}\left|W_{1}\left(\chi_{1}, \lambda\right) W_{2}\left(\chi_{2}, \lambda\right) W_{3}\left(\chi_{3}, \lambda\right)\right| d \lambda .
\end{aligned}
$$

Without loss of generality, suppose $R_{1} \geq R_{2}, R_{3}$. In the integral in $I_{3}\left(R_{1}, R_{2}, R_{3}\right)$, we take out $\left|W_{1}\left(\chi_{1}, \lambda\right)\right|$ and then use Schwarz's inequality to get

$$
\begin{aligned}
& \left|I_{3}\left(R_{1}, R_{2}, R_{3}\right)\right| \\
& \ll \sum_{r_{1} \sim R_{1}} \sum_{\chi_{1}}^{*} \max _{|\lambda|} r_{1}\left|W_{1 /\left(r_{1} Q\right)}\right| W_{1}\left(\chi_{1}, \lambda\right) \mid \\
& \quad \times \sum_{r_{2} \sim R_{2}} \sum_{\chi_{2} \bmod r_{2}}^{*}\left\{\int_{|\lambda| \leq 1 /\left(r_{2} Q\right)}\left|W_{2}\left(\chi_{2}, \lambda\right)\right|^{2} d \lambda\right\}^{1 / 2} \\
& \quad \times \sum_{r_{3} \sim R_{3}} \frac{\sqrt{\left(\varpi, r_{0}\right)}}{r_{0}} \sum_{\chi_{3} \bmod r_{3}}^{*}\left\{\int_{|\lambda| \leq 1 /\left(r_{3} Q\right)}\left|W_{3}\left(\chi_{3}, \lambda\right)\right|^{2} d \lambda\right\}^{1 / 2} .
\end{aligned}
$$

We now consider two cases.

CASE (I): $R_{1} \gg N^{1 / 10}$. The innermost sum over $r_{3}$ in $I_{3}\left(R_{1}, R_{2}, R_{3}\right)$ is $K_{3}\left(\left[r_{1}, r_{2}\right], R_{3}\right)$. Applying the bound in Lemma $3.2(\mathrm{i})$ twice then yields

$$
\begin{aligned}
& I_{3}\left(R_{1}, R_{2}, R_{3}\right) \\
& \quad \ll \sum_{r_{1} \sim R_{1}} \sum_{\chi_{1} \bmod r_{1}}^{*} \max _{|\lambda| \leq 1 /\left(r_{1} Q\right)}\left|W_{1}\left(\chi_{1}, \lambda\right)\right| \\
& \quad \times \sum_{r_{2} \sim R_{2}} \sum_{\chi_{2} \bmod r_{2}}^{*}\left\{\int_{|\lambda| \leq 1 /\left(r_{2} Q\right)}\left|W_{2}\left(\chi_{2}, \lambda\right)\right|^{2} d \lambda\right\}^{1 / 2} \\
& \quad \times \frac{\sqrt{\left(\varpi,\left[r_{1}, r_{2}\right]\right)}}{\left[r_{1}, r_{2}\right]} \tau(\varpi) \tau\left(\left[r_{1}, r_{2}\right]\right) \frac{\sqrt{N}}{\left|a_{3}\right|} L^{c} \\
& \ll \\
& \ll(\varpi) R_{1}^{\varepsilon} \frac{\sqrt{N}}{\left|a_{3}\right|} \sum_{r_{1} \sim R_{1}} \sum_{\chi_{1} \bmod r_{1}|\lambda| \leq 1 /\left(r_{1} Q\right)}^{*}\left|W_{1}\left(\chi_{1}, \lambda\right)\right| K_{2}\left(r_{1}, R_{2}\right) \\
& \ll \tau^{2}(\varpi) R_{1}^{\varepsilon} \frac{N}{\left|a_{2} a_{3}\right|} \sum_{r_{1} \sim R_{1}} \frac{\sqrt{\left(\varpi, r_{1}\right)}}{r_{1}} \sum_{\chi_{1} \bmod r_{1}}^{* \lambda \mid \leq 1 /\left(r_{1} Q\right)} \max _{1}\left|W_{1}\left(\chi_{1}, \lambda\right)\right| .
\end{aligned}
$$

In the above, we have used the fact that $\tau\left(\left[r_{1}, r_{2}\right]\right) \ll\left[r_{1}, r_{2}\right]^{\varepsilon} \ll R_{1}^{\varepsilon}$. The last double sum above is $J_{1}\left(R_{1}\right)$, which we estimate by the bound 
in Lemma 3.3(i). This leads to

$$
I_{3}\left(R_{1}, R_{2}, R_{3}\right) \ll \tau^{3}(\varpi) R_{1}^{\varepsilon-1 / 4} \frac{N^{2}}{\left|a_{1} a_{2} a_{3}\right|} \ll \frac{N^{2-\varepsilon}}{\left|a_{1} a_{2} a_{3}\right|},
$$

in view of $\tau(\varpi) \ll \varpi^{\varepsilon} \ll N^{\varepsilon}$ and our assumption that $R_{1} \gg N^{1 / 10}$ in this case.

CASE (II): $R_{1} \ll N^{1 / 10}$. The procedure here is the same as in Case (I), except that we use the alternative bounds in Lemmas 3.2, 3.3 in which $\tau(\varpi)$ does not appear. In this way, we get

$$
I_{3}\left(R_{1}, R_{2}, R_{3}\right) \ll \frac{N^{2}}{\left|a_{1} a_{2} a_{3}\right| L^{c_{3}}}
$$

for any constant $c_{3}>0$.

Inserting these two cases into (4.5) and then into (4.3), we obtain

$$
I_{3} \ll \frac{N^{2}}{\left|a_{1} a_{2} a_{3}\right| L},
$$

as desired.

The treatment for the terms in $I_{2}$ is similar. For instance, the contribution of $U_{1} T_{2} U_{3}$ is

$$
\begin{aligned}
& \ll L^{3} \sum_{r_{1} \leq P} \sum_{r_{3} \leq P} \frac{\sqrt{\left(\varpi, r^{\prime}\right)}}{r^{\prime}} \\
& \times \sum_{\chi_{1} \bmod r_{1}}^{*} \sum_{\chi_{3} \bmod r_{3}}^{*} \int_{|\lambda| \leq 1 /\left(r^{\prime} Q\right)}\left|W_{1}\left(\chi_{1}, \lambda\right) V_{2}(\lambda) W_{3}\left(\chi_{3}, \lambda\right)\right| d \lambda \\
& \ll L^{5} \max _{R_{1}, R_{3} \leq P} \sum_{r_{1} \sim R_{1}} \sum_{r_{3} \sim R_{3}} \frac{\sqrt{\left(\varpi, r^{\prime}\right)}}{r^{\prime}} \sum_{\chi_{1} \bmod r_{1}}^{*} \sum_{\chi_{3} \bmod r_{3}}^{*} \\
& \quad \times \int_{|\lambda| \leq 1 /\left(r^{\prime} Q\right)}\left|W_{1}\left(\chi_{1}, \lambda\right) V_{2}(\lambda) W_{3}\left(\chi_{3}, \lambda\right)\right| d \lambda,
\end{aligned}
$$

where $r^{\prime}=\left[r_{1}, r_{3}\right]$. Without loss of generality, assume $R_{1} \geq R_{3}$. Then the inner integral is

$$
\begin{aligned}
& \leq \max _{|\lambda| \leq 1 /\left(r_{1} Q\right)}\left|W_{1}\left(\chi_{1}, \lambda\right)\right|\left\{\int_{|\lambda| \leq 1 / Q}\left|V_{2}(\lambda)\right|^{2} d \lambda\right\}^{1 / 2} \\
& \quad \times\left\{\int_{|\lambda| \leq 1 /\left(r_{3} Q\right)}\left|W_{3}\left(\chi_{3}, \lambda\right)\right|^{2} d \lambda\right\}^{1 / 2} .
\end{aligned}
$$

By Lemma 4.1(i) (note that $1 / Q<1 /\left|2 a_{2}\right|$ ) we see that the right hand side 
of (4.7) is

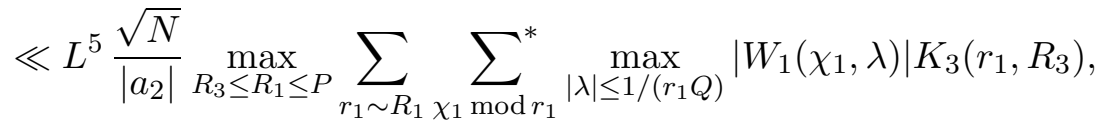

which can be handled as before by considering separately the cases $R_{1} \ll$ $N^{1 / 10}$ and $R_{1} \gg N^{1 / 10}$.

The treatment of the three terms in $I_{1}$ is even simpler, requiring only Lemma 4.1(i) twice and Lemma 3.3. This completes the proof of Theorem 2.1.

5. Bounds for $K_{j}$ and $J_{j}$. Let

$$
\widehat{W}_{j}(\chi, \lambda)=\sum_{M<\left|a_{j}\right| m \leq N}\left(\Lambda(m) \chi(m)-\delta_{\chi}\right) e\left(a_{j} m \lambda\right) .
$$

Then

$$
\begin{aligned}
& W_{j}(\chi, \lambda)-\widehat{W}_{j}(\chi, \lambda) \\
& \quad=-\sum_{k \geq 2} \sum_{M<\left|a_{j}\right| p^{k} \leq N}(\log p) \chi(p) e\left(a_{j} p^{k} \lambda\right) \ll \sqrt{N_{j}} .
\end{aligned}
$$

The contributions of this error term $\sqrt{N_{j}}$ to $J_{j}$ and $K_{j}$ are

$$
\ll \sqrt{N_{j}} \sum_{r \sim R} \sqrt{(\varpi, r)} \text { and } \sqrt{\frac{R N_{j}}{Q}} \sum_{r \sim R} \frac{\sqrt{(\varpi,[g, r])}}{[g, r]}
$$

respectively. Estimating these by using (5.8), (5.9), (5.20) and (5.22) below, we see that these are negligible in comparison with our bounds for $J_{j}$ and $K_{j}$. We shall henceforth replace $W_{j}$ by $\widehat{W}_{j}$ in $J_{j}$ and $K_{j}$.

Proof of Lemma 3.3. To the sum

$$
\sum_{M_{j}<m \leq u} \Lambda(m) \chi(m), \quad u \leq N_{j}
$$

in $J_{j}$, we apply Heath-Brown's identity (see Lemma 1 in [7]) with $k=5$, which states that

$$
\Lambda(m)=\sum_{j=1}^{5}\left(\begin{array}{l}
5 \\
j
\end{array}\right)(-1)^{j-1} \sum_{\substack{m_{1} \cdots m_{2 j}=m \\
m_{j+1}, \ldots, m_{2 j} \leq u^{1 / 5}}}\left(\log m_{1}\right) \mu\left(m_{j+1}\right) \cdots \mu\left(m_{2 j}\right) .
$$

Dividing the summation range for each $m_{i}$ into dyadic intervals of the form $\left(D_{i}, 2 D_{i}\right]$, where $D_{1}, \ldots, D_{10}$ satisfy the conditions in (3.6) with $Y=M_{j}$ 
and $X=N_{j}$, we see that the sum in (5.2) is equal to $\sum_{\mathbf{D}} \sigma(u ; \mathbf{D})$ with

$$
\sigma(u ; \mathbf{D}):=\sum_{\substack{m_{1} \sim D_{1} \\ M_{j}<m_{1} \cdots m_{10} \leq u}} \cdots \sum_{\substack{m_{10} \sim D_{10} \\ \text { in }}} b_{1}\left(m_{1}\right) \chi\left(m_{1}\right) \cdots b_{10}\left(m_{10}\right) \chi\left(m_{10}\right) .
$$

Here the functions $b_{i}$ are defined in (3.7) and $\sum_{\mathbf{D}}$ is the summation over all the vectors $\mathbf{D}=\left(D_{1}, \ldots, D_{10}\right)$ which satisfy (3.6). By the definition of $F_{\mathbf{D}}(s, \chi)$ in (3.8) and by using Perron's summation formula (see, for example, Lemma 3.12 in [14]), we have

$$
\sigma(u ; \mathbf{D})=\frac{1}{2 \pi i} \int_{1+1 / L-i T}^{1+1 / L+i T} F(s, \chi) \frac{u^{s}-M_{j}^{s}}{s} d s+O\left(L^{2}\right)
$$

where $T=N_{j}$.

As usual, we shift the path of integration to the vertical line $\operatorname{Re}(s)=1 / 2$ (note that $F_{\mathbf{D}}(s, \chi)$ is a Dirichlet polynomial and hence has no poles) and estimate the contributions on the two horizontal segments as

$$
\max _{1 / 2 \leq \sigma \leq 1+1 / L}\left|F_{\mathbf{D}}(\sigma \pm i T, \chi)\right| \frac{u^{\sigma}}{T} \ll \max _{1 / 2 \leq \sigma \leq 1+1 / L} N_{j}^{1-\sigma} L \frac{u^{\sigma}}{T} \ll L,
$$

on using the trivial estimate

$$
\begin{aligned}
F_{\mathbf{D}}(\sigma \pm i T, \chi) & \ll\left|f_{1}(\sigma \pm i T, \chi)\right| \cdots\left|f_{10}(\sigma \pm i T, \chi)\right| \\
& \ll\left(D_{1}^{1-\sigma} L\right) D_{2}^{1-\sigma} \cdots D_{10}^{1-\sigma} \ll N_{j}^{1-\sigma} L .
\end{aligned}
$$

Then we find that

$$
\sigma(u ; \mathbf{D})=\frac{1}{2 \pi} \int_{-T}^{T} F_{\mathbf{D}}\left(\frac{1}{2}+i t, \chi\right) \frac{u^{1 / 2+i t}-M_{j}^{1 / 2+i t}}{1 / 2+i t} d t+O\left(L^{2}\right) .
$$

We may assume that $R \geq 1$ so that the primitive character $\chi \bmod r$ is not principal and $\delta_{\chi}=0$. Then

$$
\begin{aligned}
\widehat{W}_{j}(\chi, \lambda)= & \sum_{M_{j}<m \leq N_{j}} \Lambda(m) \chi(m) e\left(a_{j} m \lambda\right)=\sum_{\mathbf{D}} \int_{M_{j}}^{N_{j}} e\left(a_{j} u \lambda\right) d(\sigma(u ; \mathbf{D})) \\
= & \sum_{\mathbf{D}} \frac{1}{2 \pi} \int_{-T}^{T} F_{\mathbf{D}}\left(\frac{1}{2}+i t, \chi\right) \int_{M_{j}}^{N_{j}} u^{-1 / 2+i t} e\left(a_{j} u \lambda\right) d u d t \\
& +O\left\{(1+|\lambda| N) L^{12}\right\} .
\end{aligned}
$$

The inner integral over $u$ is equal to

$$
\int_{M_{j}}^{N_{j}} u^{-1 / 2} e\left(\frac{t}{2 \pi} \log u+a_{j} \lambda u\right) d u
$$


which we now estimate by means of Lemmas 4.3 and 4.5 of [14]. Let $T_{0}=$ $4 \pi N /(R Q)=4 \pi P L / R$. Since

$$
\left|\frac{d}{d u}\left(\frac{t}{2 \pi} \log u+a_{j} \lambda u\right)\right|=\left|\frac{t}{2 \pi u}+a_{j} \lambda\right| \geq \frac{|t|}{4 \pi N_{j}}
$$

for $|t|>T_{0}$, and

we have

$$
\left|\frac{d^{2}}{d u^{2}}\left(\frac{t}{2 \pi} \log u+a_{j} \lambda u\right)\right|=\left|-\frac{t}{2 \pi u^{2}}\right| \geq \frac{|t|}{2 \pi N_{j}^{2}},
$$

$$
\begin{aligned}
& \int_{M_{j}}^{N_{j}} u^{-1 / 2} e\left(\frac{t}{2 \pi} \log u+a_{j} \lambda u\right) d u \\
& \qquad \begin{cases}\sqrt{N_{j}} / \sqrt{|t|+1} & \text { for }|t| \leq T_{0}, \\
\sqrt{N_{j}} /|t| & \text { for } T_{0}<|t| \leq T .\end{cases}
\end{aligned}
$$

Therefore,

$$
\begin{aligned}
\max _{|\lambda| \leq 1 /(r Q)}\left|\widehat{W}_{j}(\chi, \lambda)\right| \ll & \sqrt{N_{j}} \sum_{\mathbf{D}}\left\{\int_{|t| \leq T_{0}}\left|F_{\mathbf{D}}\left(\frac{1}{2}+i t, \chi\right)\right| \frac{d t}{\sqrt{|t|+1}}\right. \\
& \left.+\int_{T_{0}<|t| \leq N_{j}}\left|F_{\mathbf{D}}\left(\frac{1}{2}+i t, \chi\right)\right| \frac{d t}{|t|}\right\}+\frac{N L^{12}}{R Q} .
\end{aligned}
$$

Thus,

$$
\begin{aligned}
& \sum_{\substack{r \sim R \\
d \mid r}} \sum_{\chi \bmod r}^{*} \max _{|\lambda| \leq 1 /(r Q)}\left|\widehat{W}_{j}(\chi, \lambda)\right| \\
& \ll \sqrt{N_{j}} L \sum_{\mathbf{D}}\left\{\max _{Y \leq T_{0}} \frac{1}{\sqrt{Y}} \sum_{\substack{r \sim R \\
d \mid r}} \sum_{\chi \bmod r}^{*} \int_{|t| \sim Y}\left|F_{\mathbf{D}}\left(\frac{1}{2}+i t, \chi\right)\right| d t\right. \\
& \left.\quad+\max _{T_{0}<Z \leq N_{j}} \frac{1}{Z} \sum_{\substack{r \sim R \\
d \mid r}} \sum_{\chi \bmod r}^{*} \int_{|t| \sim Z}\left|F_{\mathbf{D}}\left(\frac{1}{2}+i t, \chi\right)\right| d t\right\}+\frac{N R}{Q d} L^{12} .
\end{aligned}
$$

Applying now Lemma 3.4 with $X=N_{j}$, the above is

$$
\ll\left(\frac{R^{2}}{d} \sqrt{T_{0} N_{j}}+\frac{R}{\sqrt{d}} N_{j}^{4 / 5}+N_{j}\right) L^{c} .
$$

Notice that for any function $H(r)$,

$$
\sum_{r \sim R} \frac{\sqrt{(\varpi, r)}}{r} H(r) \leq \frac{1}{R} \sum_{\substack{d \mid \varpi \\ d \ll R}} \sqrt{d} \sum_{\substack{r \sim R \\ d \mid r}}|H(r)| .
$$


Hence by (5.7) and the definition of $J_{j}(R)$ in (3.4),

$$
\begin{aligned}
J_{j}(R) & \ll \frac{1}{R} \sum_{\substack{d \mid \varpi \\
d \ll R}}\left(\sqrt{\frac{T_{0} N_{j}}{d}} R^{2}+R N_{j}^{4 / 5}+\sqrt{d} N_{j}\right) L^{c} \\
& \ll \tau(\varpi)\left(\sqrt{R P N_{j}}+N_{j}^{4 / 5}+R^{-1 / 2} N_{j}\right) L^{c} .
\end{aligned}
$$

In view of the assumption that $R \ll P \leq N_{j}^{2 / 5}$, this yields the bound in Lemma 3.3(i).

To prove the alternative bound for $J_{j}(R)$ in Lemma 3.3(ii), we note trivially that

$$
r^{-1} \sqrt{(\varpi, r)} \leq r^{-1 / 2}
$$

Hence by (5.7) and definition (3.4),

$$
\begin{aligned}
J_{j}(R) & \ll R^{-1 / 2}\left\{R^{2} \sqrt{T_{0} N_{j}}+R N_{j}^{4 / 5}+N_{j}\right\} L^{c} \\
& \ll\left\{R \sqrt{P N_{j}}+\sqrt{R} N_{j}^{4 / 5}+R^{-1 / 2} N_{j}\right\} L^{c} .
\end{aligned}
$$

This yields the desired bound in Lemma 3.3(ii) provided (in addition to the condition $R \ll N^{1 / 10}$ ) that $R$ is greater than a sufficiently large power of $L$.

It remains, therefore, to consider the situation when $R \ll L^{c_{4}}$ for any large constant $c_{4}$. In this case we shall obtain the bound

$$
J_{j}(R) \ll \frac{N}{\left|a_{j}\right|} \exp \left\{-c L^{1 / 5}\right\}
$$

which is good enough.

We begin with the explicit formula (see [4, pp. 109 and 120])

$$
\sum_{m \leq u} \Lambda(m) \chi(m)=\delta_{\chi} u-\sum_{|\gamma| \leq T} \frac{u^{\varrho}}{\varrho}+O\left\{\left(\frac{u}{T}+1\right) \log ^{2}(r u T)\right\},
$$

where $\varrho=\beta+i \gamma$ is a typical non-trivial zero of the function $L(s, \chi)$ and $T$ is a parameter satisfying $2 \leq T \leq u$. Taking $T=M_{j}$ in (5.11) and then inserting it into $\widehat{W}_{j}(\chi, \lambda)$, we get

$$
\begin{aligned}
\widehat{W}_{j}(\chi, \lambda) & =\int_{M_{j}}^{N_{j}} e\left(a_{j} u \lambda\right) d\left\{\sum_{n \leq u}\left(\Lambda(m) \chi(m)-\delta_{\chi}\right)\right\} \\
& =-\sum_{|\gamma| \leq T} \int_{M_{j}}^{N_{j}} u^{\varrho-1} e\left(a_{j} u \lambda\right) d u+O\left\{(1+|\lambda| N) L^{2}\right\} .
\end{aligned}
$$


The last integral is bounded in the same way as in (5.4) and we have

$$
\begin{aligned}
\int_{M_{j}}^{N_{j}} u^{\varrho-1} e\left(a_{j} u \lambda\right) d u & =\int_{M_{j}}^{N_{j}} u^{\beta-1} e\left(\frac{\gamma}{2 \pi} \log u+a_{j} \lambda u\right) d u \\
& \ll \begin{cases}N_{j}^{\beta} / \sqrt{|\gamma|+1} & \text { if }|\gamma| \leq T_{0}, \\
N_{j}^{\beta} /|\gamma| & \text { if } T_{0}<|\gamma| \leq T,\end{cases}
\end{aligned}
$$

where, as before, $T_{0}=4 \pi N /(R Q)$. Applying this to (5.12) we have

$$
\begin{aligned}
J_{j}(R) \ll & \sum_{r \sim R} \sum_{\chi \bmod r}^{*} \max _{|\lambda| \leq 1 /(r Q)}\left|\widehat{W}_{j}(\chi, \lambda)\right| \\
\ll & \sum_{r \sim R} \sum_{\chi \bmod r}^{*} \sum_{|\gamma| \leq T_{0}} \frac{N_{j}^{\beta}}{\sqrt{|\gamma|+1}} \\
& +\sum_{r \sim R} \sum_{\chi \bmod r}^{*} \sum_{T_{0}<|\gamma| \leq N_{j}} \frac{N_{j}^{\beta}}{|\gamma|}+R P L^{2} \\
= & : \sum_{r \sim R} H_{1}+\sum_{r \sim R} H_{2}+R P L^{2},
\end{aligned}
$$

say. The last term above is clearly acceptable.

Vinogradov's zero-free region (see Satz VIII.6.2 in Prachar [13]) states that for any $\chi \bmod r$, there exists a constant $c_{5}>0$ such that $L(\sigma+i t, \chi) \neq 0$ in the region

$$
\sigma \geq 1-\frac{c_{5}}{\log r+\log ^{4 / 5}(|t|+2)}
$$

except for the possible Siegel zero. However, for $r \ll L^{c_{4}}$ the Siegel zero does not exist. It follows that $L(s, \chi)$ is zero-free for $\sigma \geq 1-\eta(\tau)$ and $|t| \leq \tau$, where $\eta(\tau)=c_{5} /\left(2 \log ^{4 / 5} \tau\right)$. Consequently, the inner sum in $H_{2}$ is

$$
\ll N_{j}^{1-\eta\left(N_{j}\right)} \sum_{|\gamma| \leq N_{j}} \frac{1}{|\gamma|} \ll N_{j} \exp \left\{-\frac{c_{5}}{3} \log ^{1 / 5} N_{j}\right\},
$$

and

$$
H_{2} \ll N_{j} \exp \left\{-\frac{c_{5}}{4} \log ^{1 / 5} N\right\} .
$$

Recall that $R \ll L^{c_{4}}$, so the contribution of $H_{2}$ to $J_{j}(R)$ is acceptable.

Finally, we bound the remaining sum involving $H_{1}$, by using the zerodensity theorem that 


$$
\sum_{\chi \bmod r} N(\chi, \tau) \ll(r \tau)^{3(1-\sigma) /(2-\sigma)}(\log r \tau)^{9},
$$

where $N(\chi, \tau)$ denotes the number of zeros $\varrho=\beta+i \gamma$ of $L(s, \chi)$ with $\sigma \leq \beta \leq 1,|\gamma| \leq \tau$. Then

$$
\begin{aligned}
H_{1} & \ll L \max _{Z \leq T_{0}} \frac{1}{\sqrt{Z}} \sum_{\chi \bmod r} \sum_{|\gamma| \sim Z} N_{j}^{\beta} \\
& \ll L^{10} \max _{Z \leq T_{0}} \frac{1}{\sqrt{Z}} \int_{1 / 2}^{1-\eta(Z)} N_{j}^{\sigma}(r Z)^{3(1-\sigma) /(2-\sigma)} d \sigma,
\end{aligned}
$$

by Stieltjes integration. The exponent of $Z$ here is

$$
\phi(\sigma)=\frac{3(1-\sigma)}{2-\sigma}-\frac{1}{2},
$$

which is positive when $\sigma<4 / 5$ and is negative when $\sigma>4 / 5$. Thus, by dividing the above integral at the point $4 / 5$, we have

$$
H_{1} \ll L^{c} \int_{1 / 2}^{4 / 5} N_{j}^{\sigma} T_{0}^{\phi(\sigma)} d \sigma+L^{c} \int_{4 / 5}^{1-\eta\left(T_{0}\right)} N_{j}^{\sigma} d \sigma .
$$

The second term here, by definition of $T_{0}$, is

$$
\ll L^{c} N_{j}^{1-\eta\left(T_{0}\right)} \ll N_{j} \exp \left\{-\frac{c_{5}}{10} \log ^{1 / 5} N_{j}\right\},
$$

which is good enough. For the first integral in (5.14), since $T_{0} \ll P \leq N_{j}^{2 / 5}$, it is

$$
\ll \int_{1 / 2}^{4 / 5} N_{j}^{\sigma} P^{\phi(\sigma)} d \sigma \leq \int_{1 / 2}^{4 / 5} N_{j}^{\phi_{1}(\sigma)} d \sigma
$$

where

$$
\phi_{1}(\sigma)=\sigma-\frac{1}{5}+\frac{6(1-\sigma)}{5(2-\sigma)} .
$$

The maximum value of $\phi_{1}(\sigma)$ for $\sigma \in[1 / 2,4 / 5]$ is $\phi_{1}(4 / 5)=4 / 5$. This leads to the bound $N_{j}^{4 / 5}$ for the integral in (5.15). In view of (5.13) and the assumption that $R \ll L^{c_{4}}$, the desired bound (5.10) follows. This finishes the proof of Lemma 3.3.

We now come to prove the bounds for $K_{j}$ in Lemma 3.2.

Proof of Lemma 3.2. First, by Gallagher's lemma (see [5, Lemma 1]), we have 


$$
\begin{aligned}
& \int_{|\lambda| \leq 1 /(r Q)}\left|\widehat{W}_{j}(\chi, \lambda)\right|^{2} d \lambda \\
& \ll\left(\frac{1}{r Q}\right)^{2} \int_{-\infty}^{\infty}\left|\sum_{\substack{v<\left|a_{j}\right| m \leq v+r Q \\
M<\left|a_{j}\right| m \leq N}}\left(\Lambda(m) \chi(m)-\delta_{\chi}\right)\right|^{2} d v \\
& \ll\left(\frac{1}{r Q}\right)^{2} \int_{M-r Q}^{N}\left|\sum_{\substack{v<\left|a_{j}\right| m \leq v+r Q \\
M<\left|a_{j}\right| m \leq N}}\left(\Lambda(m) \chi(m)-\delta_{\chi}\right)\right|^{2} d v
\end{aligned}
$$

Thus, in view of the definition (3.5),

$$
\begin{aligned}
K_{j}(g, R) \ll & \sqrt{N} \sum_{r \sim R} \frac{\sqrt{(\varpi,[g, r])}}{[g, r]} \frac{1}{r Q} \\
& \times \sum_{\chi \bmod r}^{*} \max _{I_{r}}\left|\sum_{m \in I_{r}}\left(\Lambda(m) \chi(m)-\delta_{\chi}\right)\right|,
\end{aligned}
$$

where the maximum is over all intervals $I_{r}$ lying in $\left[M_{j}, N_{j}\right]$ of length $\ll r Q\left|a_{j}\right|^{-1}$. For the sum $\sum_{m \in I_{r}}$ in (5.17), we apply Heath-Brown's identity in the same way as for $J_{j}$ in (5.2)-(5.3). If we write $I_{r}=(Y, X]$ where $M_{j} \leq Y<X \leq N_{j}$, then as in (5.3),

$$
\begin{aligned}
\sum_{m \in I_{r}} \Lambda(m) \chi(m) & =\sum_{\mathbf{D}} \sigma(u ; \mathbf{D}) \\
& =\frac{1}{2 \pi} \sum_{\mathbf{D}} \int_{|t| \leq N_{j}} F_{\mathbf{D}}\left(\frac{1}{2}+i t, \chi\right) \frac{X^{1 / 2+i t}-Y^{1 / 2+i t}}{1 / 2+i t} d t+O\left(L^{12}\right) .
\end{aligned}
$$

The factor $\left(X^{1 / 2+i t}-Y^{1 / 2+i t}\right)(1 / 2+i t)^{-1}$ inside the integral is clearly $\ll \sqrt{N_{j}} /|t|$ for $|t|>T_{0}$ and is

$$
\ll\left|\int_{Y}^{X} u^{-1 / 2+i t} d u\right| \ll \frac{X-Y}{\sqrt{N_{j}}} \ll \frac{r Q}{\sqrt{N_{j}}\left|a_{j}\right|}
$$

for $|t| \leq T_{0}$. Here $T_{0}=N /(R Q)$ is the same as before. Therefore,

$$
\begin{aligned}
\max _{I_{r}}\left|\sum_{m \in I_{r}}\left(\Lambda(m) \chi(m)-\delta_{\chi}\right)\right| & \\
\ll & \sum_{\mathbf{D}} \frac{r Q}{\sqrt{\left|a_{j}\right| N}} \int_{|t| \leq T_{0}}\left|F_{\mathbf{D}}\left(\frac{1}{2}+i t, \chi\right)\right| d t \\
& +\sum_{\mathbf{D}} \sqrt{N_{j}} \int_{T_{0}<|t| \leq N_{j}}\left|F_{\mathbf{D}}\left(\frac{1}{2}+i t, \chi\right)\right| \frac{d t}{|t|}+L^{12} .
\end{aligned}
$$


Similarly to (5.9) and applying Lemma 3.4, we have

$$
\sum_{\substack{r \sim R \\ d \mid r}} \sum_{\chi \bmod r}^{*} \max _{I_{r}}\left|\sum_{m \in I_{r}}\left(\Lambda(m) \chi(m)-\delta_{\chi}\right)\right|
$$

$\ll \sum_{\mathbf{D}} \frac{R Q}{\sqrt{\left|a_{j}\right| N}} \sum_{\substack{r \sim R \\ d \mid r}} \sum_{\chi \bmod r}^{*} \int_{|t| \leq T_{0}}\left|F_{\mathbf{D}}\left(\frac{1}{2}+i t, \chi\right)\right| d t$

$$
+\sum_{\mathbf{D}} \sqrt{N_{j}} L \max _{T_{0}<Z \leq N_{j}} \frac{1}{Z} \sum_{\substack{r \sim R \\ d \mid r}} \sum_{\chi \bmod r}^{*} \int_{|t| \sim Z}\left|F_{\mathbf{D}}\left(\frac{1}{2}+i t, \chi\right)\right| d t+\frac{R^{2}}{d} L^{12}
$$

$\ll L^{11} \sqrt{N_{j}}\left(\frac{R^{2}}{d}+\frac{R}{\sqrt{d T_{0}}} N_{j}^{3 / 10}+\frac{\sqrt{N_{j}}}{T_{0}}\right)$.

To prove Lemma 3.2(i), we observe that

$$
\begin{aligned}
\frac{\sqrt{(\varpi,[g, r])}}{[g, r]} & =\frac{(g, r)}{g r} \sqrt{\left(\varpi, g \frac{r}{(g, r)}\right)} \\
& \leq \frac{\sqrt{(\varpi, g)}}{g} \frac{(g, r)}{r} \sqrt{\left(\varpi, \frac{r}{(g, r)}\right)}
\end{aligned}
$$

and hence, for any function $H(r)$,

$$
\sum_{r \sim R} \frac{\sqrt{(\varpi,[g, r])}}{[g, r]}|H(r)| \ll \frac{\sqrt{(\varpi, g)}}{g R} \sum_{\substack{h|g \\ k| \varpi}} h \sqrt{k} \sum_{\substack{r \sim R \\ h k \mid r}}|H(r)| .
$$

Using this and (5.18) we deduce from (5.17) that

$$
\begin{aligned}
& K_{j}(g, R) \\
& \ll \frac{\sqrt{(\varpi, g)}}{g} L^{11} \frac{\sqrt{N N_{j}}}{R^{2} Q} \sum_{\substack{h|g, k| \varpi \\
h k \leq 2 R}} h \sqrt{k}\left(\frac{R^{2}}{h k}+\frac{R}{\sqrt{h k T_{0}}} N_{j}^{3 / 10}+\frac{\sqrt{N_{j}}}{T_{0}}\right) \\
& \ll \frac{\sqrt{(\varpi, g)}}{g} L^{c} \tau(g) \tau(\varpi) \frac{P}{R^{2} \sqrt{\left|a_{j}\right|}}\left(R^{2}+\frac{R^{3 / 2}}{\sqrt{T_{0}}} N_{j}^{3 / 10}+R \frac{\sqrt{N_{j}}}{T_{0}}\right) \\
& \ll \frac{\sqrt{(\varpi, g)}}{g} L^{c} \tau(g) \tau(\varpi)\left(\frac{P}{\sqrt{\left|a_{j}\right|}}+\frac{\sqrt{P}}{\sqrt{\left|a_{j}\right|}} N_{j}^{3 / 10}+\frac{\sqrt{N}}{\left|a_{j}\right|}\right) \\
& \ll \frac{\sqrt{(\varpi, g)}}{g} L^{c} \tau(g) \tau(\varpi) \frac{\sqrt{N}}{\left|a_{j}\right|},
\end{aligned}
$$


on recalling that $T_{0}=N /(R Q)$ and $P=(N / A)^{2 / 5} \leq N_{j}^{2 / 5}$. This proves the bound in Lemma 3.2(i).

To prove the bound in (ii), it is sufficient to use, instead of (5.20), the cruder inequality

$$
\begin{aligned}
\sum_{r \sim R} \frac{\sqrt{(\varpi,[g, r])}}{[g, r]}|H(r)| & \leq \frac{\sqrt{(\varpi, g)}}{g} \sum_{r \sim R} \frac{(g, r)}{r} \sqrt{\left(\varpi, \frac{r}{(g, r)}\right)}|H(r)| \\
& =\frac{\sqrt{(\varpi, g)}}{g} \sum_{r \sim R} \frac{\sqrt{(g, r)}|H(r)|}{\sqrt{r}} \\
& \ll \frac{\sqrt{(\varpi, g)}}{g \sqrt{R}} \sum_{\substack{d \mid g \\
d \leq 2 R} \substack{r \sim R \\
d \mid r}} \sqrt{d} \sum_{\substack{r \sim 2 \\
d}}|H(r)|
\end{aligned}
$$

for any function $H(r)$. So, parallel to the deduction of (5.21), we have

$$
\begin{aligned}
& K_{j}(g, R) \\
& \ll \frac{\sqrt{(\varpi, g)}}{g} L^{11} \sum_{\substack{d \mid g \\
d \leq 2 R}} \sqrt{d} \frac{\sqrt{N_{j} N}}{R^{3 / 2} Q}\left(\frac{R^{2}}{d}+\frac{R}{\sqrt{T_{0} d}} N_{j}^{3 / 10}+\frac{\sqrt{N_{j}}}{T_{0}}\right) \\
& \ll \frac{\sqrt{(\varpi, g)}}{g} L^{c} \tau(g) \frac{\sqrt{N_{j} N}}{R^{3 / 2} Q}\left(R^{2}+\frac{R}{\sqrt{T_{0}}} N_{j}^{3 / 10}+\frac{\sqrt{R N_{j}}}{T_{0}}\right) \\
& \ll \frac{\sqrt{(\varpi, g)}}{g} L^{c} \tau(g)\left(\frac{P \sqrt{R}}{\sqrt{\left|a_{j}\right|}}+\frac{\sqrt{P}}{\sqrt{\left|a_{j}\right|}} N_{j}^{3 / 10}+\sqrt{\frac{N_{j}}{\left|a_{j}\right|}}\right) .
\end{aligned}
$$

Since $P \leq N_{j}^{2 / 5}$ and $R \ll N_{j}^{1 / 10}$, the above yields the bound in Lemma 3.2(ii). This completes our proof of Lemma 3.2.

\section{References}

[1] A. Baker, On some Diophantine inequalities involving primes, J. Reine Angew. Math. 228 (1967), 166-181.

[2] K. K. Choi, A numerical bound for Baker's constant-some explicit estimates for small prime solutions of linear equations, Bull. Hong Kong Math. Soc. 1 (1997), $1-19$.

[3] K. K. Choi, M. C. Liu, and K. M. Tsang, Conditional bounds for small prime solutions of linear equations, Manuscripta Math. 74 (1992), 321-340.

[4] H. Davenport, Multiplicative Number Theory, 2nd ed., Springer, Berlin, 1980.

[5] P. X. Gallagher, A large sieve density estimate near $\sigma=1$, Invent. Math. 11 (1970), 329-339.

[6] H. Hasse, Vorlesungen über Zahlentheorie, 2nd ed., Grundlehren Math. Wiss. 59, Springer, Berlin, 1964. 
[7] D. R. Heath-Brown, Prime numbers in short intervals and a generalized Vaughan identity, Canad. J. Math. 34 (1982), 1365-1377.

[8] H. Z. Li, Small prime solutions of some ternary linear equations, Acta Arith. 98 (2001), 293-309.

[9] J. Y. Liu, On Lagrange's theorem with prime variables, Quart. J. Math. 54 (2003), 453-462.

[10] M. C. Liu and K. M. Tsang, Small prime solutions of linear equations, in: Théorie des nombres, J.-M. De Koninck and C. Levesque (eds.), de Gruyter, Berlin, 1989, 595-624.

[11] - - - On pairs of linear equations in three prime variables and an application to Goldbach's problem, J. Reine Angew. Math. 399 (1989), 109-136.

[12] M. C. Liu and T. Z. Wang, A numerical bound for small prime solutions of some ternary linear equations, Acta Arith. 86 (1998), 343-383.

[13] K. Prachar, Primzahlverteilung, Springer, Berlin, 1957.

[14] E. C. Titchmarsh, The Theory of the Riemann Zeta-Function, 2nd ed., Oxford Univ. Press, 1986.

Department of Mathematics

Shandong University

Jinan, Shandong 250100, P.R. China

E-mail: jyliu@sdu.edu.cn
Department of Mathematics The University of Hong Kong Pokfulam Road, Hong Kong E-mail: kmtsang@maths.hku.hk 